\title{
Strip Yield Model for a Crack in a Plate of Arbitrary Thickness
}

\author{
A. Kotousov ${ }^{1}$ and C. H. Wang ${ }^{2}$ \\ ${ }^{1}$ Department of Mechanical Engineering, Monash University, PO Box 31, VIC \\ 3800, Australia. \\ ${ }^{2}$ Aeronautical and Maritime Research Laboratory, Defense Science and \\ Technology Organization, 506 Lorimer Street, Fishermans Bend VIC 3207, \\ Australia.
}

\begin{abstract}
Fatigue and fracture of plate-like structures exhibit a strong dependence on the cross-sectional thickness, when the results are expressed in terms of stress-intensity factors. A theoretical approach to account for this plate-thickness effect is presented, within the framework of the generalized plane-strain theory by Kane and Mindlin. Fundamental solutions (Green's functions) for an edge dislocation in a plate of arbitrary thickness are first obtained, and then applied to analyze the effect of plate thickness on the elasto-plastic deformation behavior at the tip of a through-the-thickness tensile crack.
\end{abstract}

\section{INTRODUCTION}

For plate-like structures, the cross-sectional thickness has a strongly influence on both the fatigue crack growth rates $[1,2]$ and the fracture toughness, especially when the size of the process zone is comparable to the plate thickness. This is primarily due to significant difference between the stressstate at the tip of a through-thickness crack in a plate of arbitrary thickness and that corresponding to either the idealized plane-stress or plane-strain conditions. In the case of fatigue crack growth, this difference often manifests itself in a transition from flat to slant crack growth in thin structures [3]. For fatigue life calculations, this difference in the in-plane and out-of-plane constraints may significantly affect the accuracy of predictions [2] when the base-line fatigue crack growth curves generated using standard specimens (often under plane-strain conditions) are applied to predict the fatigue life of engineering structures of thin cross section.

Studies to date [4,5] have generally addressed separately two kinds of constraint: in-plane stresses and out-of-plane stresses. The deviation of the 
actual three-dimensional stress-state at a crack-tip from that under idealized plane-stress conditions provides a measure of the constraint. The magnitudes of these in-plane and out-of-plane constraints depend upon the cross-section thickness of the structure relative to the applied load and the material properties. Under the small scale yielding (SSY) condition, the effect of inplane constraint on fatigue crack growth is relatively weak, as confirmed by experimental observations [6] indicating that biaxial loading only moderately alter the crack growth rates. However, the specimen thickness has been found to affect fatigue crack growth rates under the same applied stress intensity factor $[1,7,8,9]$. This effect is particularly strong when crack-tip plastic zone size becomes comparable to the thickness, a problem frequently encountered in analyzing thin section structures such as aircraft skins.

Due to the complexities of the three-dimensional constraint at the tip of a through-thickness crack, earlier attempts at quantifying the effects of constraint on fatigue crack growth were based on simple assumptions such as plane-stress [10] or plane-strain conditions [11]. Accurate assessment of the thickness effect on fatigue crack growth rates and fracture toughness has relied on computational methods, such as the finite element method, where the results are to some extent dependent on the mesh density of the finite element model. Newman and his colleagues $[3,12]$ conducted detailed full three-dimensional finite element analyses for through-thickness cracks in an elastic-perfectly plastic material. From these results an approximate equation [12] for the constraint factor has been constructed, which varies with the ratio of crack-tip plastic zone to plate thickness [2,3] in the case of small-scale yielding. In the presence of large-scale yielding, no results are currently available on the appropriate value of the constraint factor.

The purpose of this paper is to present a new theoretical approach to account for the effect of cross-sectional thickness on the fracture of a plate of arbitrary thickness. The analysis is based on the generalized plane-strain theory of Kane and Mindlin [13], which is summarized in the next section.

\section{GENERALIZED PLANE STRAIN THEORY}

A plate theory, which includes the through-the-thickness stress components, while retaining the simplicity of a two-dimensional model, was introduced by 
Kane and Mindlin [13] in their work on the high-frequency extensional vibrations of plates. This theory, to be termed the generalized plane-strain theory, was employed by Yang and Freund [14] to study the three-dimensional stress distribution near the tip of a semi-infinite crack using Wiener-Hopf technique. Their results suggested that the crack-tip field asymptotically approaches that of plane-strain over a distance comparable to plate thickness. By adopting the generalized plane-strain theory, Kotousov and Wang [15] obtained an exact solution for the three-dimensional stress distributions around a circular hole, and approximate, explicit, solutions for $\mathrm{V}$-shaped notches with a circular tip.

The theory of generalized plane strain assumes that the through-thethickness extensional strain is uniform in the thickness direction. In the case of an elastic plate bounded by planes $z= \pm h$, where $x$ and $y$ represent the inplane Cartesian coordinates, the displacement field takes the following form

$$
u_{x}=u_{x}(x, y) \quad, u_{y}=u_{y}(x, y), u_{z}=\frac{z}{h} w(x, y)
$$

It is clear that the equation (1) implies that lines normal to the mid-plane of the plate in the undeformed state remain normal to the mid-plane in the deformed state and that these lines experience uniform extensional strain $w(x, y) / h$, where $w(x, y)$ is the out-of-plane displacement at the surfaces $z= \pm h$ of the plate.

Stress resultants are defined by

$$
\begin{aligned}
& \left(N_{x x}, N_{y y}, N_{z z}, N_{x y}\right)=\int_{-h}^{h}\left(\sigma_{x x}, \sigma_{y y}, \sigma_{z z}, \tau_{x y}\right) d z \\
& \left(R_{x}, R_{y}\right)=\int_{-h}^{h}\left(\tau_{z x}, \tau_{z y}\right) z d z
\end{aligned}
$$

It is seen that the $N_{x x}, N_{y y}$, and $N_{x y}$ are the usual forces per unit length, and $N_{z z}$ is $2 h$ times the average transverse normal stress. The parameter $R_{x}$ and $R_{y}$ are components of "pinching" shear, playing a role similar to that of the transverse shear force in the corresponding equilibrium equations of flexible plates. 


\section{FUNDAMENTAL SOLUTION FOR EDGE DISLOCATION}

To develop a strip-yield model for a through-the-thickness crack in a plate of arbitrary thickness, the Green's function for an edge dislocation in a plate is required. Let the z-axis be along the dislocation line. The differential equations of equilibrium for an edge dislocation deformation in an isotropic finite thickness plate can be written as

$$
\begin{aligned}
& \nabla^{2} \mathbf{u}+\frac{1}{1-2 v} \nabla \nabla \cdot \mathbf{u}+\frac{2 v}{1-2 v} \frac{1}{h} \nabla w=\boldsymbol{\tau} \times \mathbf{b} \delta(\mathbf{r}) \\
& \nabla^{2} w-\frac{6}{h} \frac{v}{1-2 v} \nabla \cdot \mathbf{u}-\frac{6}{h^{2}} \frac{1-v}{1-2 v} w=0
\end{aligned}
$$

where the operators $\nabla$ and $\nabla^{2}$ are defined as $\nabla \equiv \frac{\partial}{\partial x} \mathbf{i}+\frac{\partial}{\partial y} \mathbf{j}$ and $\nabla^{2} \equiv \frac{\partial^{2}}{\partial x^{2}}+\frac{\partial^{2}}{\partial y^{2}}$, parameters $E$ and $v$ denote respectively the Young's modulus and the Poisson's ratio, $\mathbf{b}$ is the Burgers vector of the dislocation, $\tau$ is the tangent vector to the dislocation line, and $\mathbf{u}$ is multi-valued function subject to the following uniqueness condition,

$\oint_{\Gamma} d \mathbf{u}=-\mathbf{b}$

where the contour $\Gamma$ traverses clockwise around the vector $\tau$.

Omitting details, the stress resultants for the edge dislocation in a finite thickness plate are found to be

$$
\begin{aligned}
& \frac{N_{r r}}{2 h}=-\frac{E b \sin \theta}{4\left(1-v^{2}\right) \pi \kappa^{2} r^{3}}\left[4 v^{2}+\left(1-v^{2}\right) \kappa^{2} r^{2}-2 v^{2} \kappa^{2} r^{2} K_{0}(\kappa r)\right.\left.-4 v^{2} \kappa r K_{1}(\kappa r)\right] \\
& \frac{N_{\theta \theta}}{2 h}=\frac{E b \sin \theta}{4\left(1-v^{2}\right) \pi \kappa^{2} r^{3}}\left[4 v^{2}-\left(1-v^{2}\right) \kappa^{2} r^{2}-2 v^{2} \kappa^{2} r^{2} K_{0}(\kappa r)\right. \\
&\left.-2\left(2+\kappa^{2} r^{2}\right) v^{2} \kappa r K_{1}(\kappa r)\right]
\end{aligned}
$$




$$
\begin{aligned}
& \frac{N_{r \theta}}{2 h}=\frac{E b \cos \theta}{4\left(1-v^{2}\right) \pi \kappa^{2} r^{3}}\left[4 v^{2}+\left(1-v^{2}\right) \kappa^{2} r^{2}-2 v^{2} \kappa^{2} r^{2} K_{0}(\kappa r)\right. \\
& \frac{N_{z z}}{2 h}=-\frac{v E \kappa b \sin \theta}{2\left(1-v^{2}\right) \pi} K_{1}(\kappa r) \\
& \frac{R_{r}}{2 h}=\frac{v \kappa^{2} h}{6 \pi \kappa r(1+v)}\left[K_{1}(\kappa r)\right] \\
& \frac{R_{\theta}}{2 h}=-\frac{v \kappa^{2} h}{6 \pi \kappa r(1+v)}\left[\frac{1}{\kappa r}+K_{0}(\kappa r) \kappa r\right] E b \sin \theta
\end{aligned}
$$

where $K_{n}(\cdot)$ are modified Bessel function of $\mathrm{n}^{\text {th }}$ order and $\kappa=\frac{1}{h} \sqrt{\frac{6}{1-v}}$

It can be shown that the present solutions recovers correctly the plane-stress solution and the plane-strain solution by taking the limit $r \rightarrow 0$ with $r / h \rightarrow 0$ and $r / h \rightarrow \infty$, respectively.

\section{D STRIP YIELD MODEL}

In the following a strip-yield model similar to Dugdale model will be presented, making use of the newly developed edge dislocation solutions presented in the previous section. Plasticity is assumed to occur within a strip directly ahead of the crack tip; the variation of the plastic zone through the plate thickness is not considered. Instead, the plastic zone size is assumed to be constant in the thickness direction. In this formulation the crack and plastic zones are represented by a distribution of displacement discontinuities, or edge dislocations. Within the plastic zone, the stresses may then be written as, adopting Tresca yield criterion,

$$
\left|N_{y y}-N_{z z}\right|=2 h \sigma_{Y S}
$$


where $\sigma_{Y S}$ denotes the material's yield stress under uniaxial tension. Thus, the equilibrium condition can be written as,

$$
\frac{E h}{2 \pi\left(1-v^{2}\right)} \int_{-a-r_{p}}^{a+r_{p}} B(t) G_{y y}(x-t) d t=-N_{y y}^{\infty} \quad, \quad|x|<a
$$

for $a \leq|x| \leq a+r_{p}$

$$
\frac{E h}{2 \pi\left(1-v^{2}\right)}\left|\int_{-a-r_{p}}^{a+r_{p}} B(t)\left[G_{y y}(x-t)-G_{z z}(x-t)\right] d t\right|=2 h \sigma_{Y S}-N_{y y}^{\infty}
$$

where the Green's function $G_{y y}$ and $G_{z z}$ is given by (5b) and (5c), referring to the stress resultants,

$$
\begin{aligned}
G_{y y}(\eta)=\frac{1}{\kappa^{2} \eta^{3}}\left[4 v^{2}-\left(1-v^{2}\right) \kappa^{2} \eta^{2}\right. & -2 v^{2} \kappa^{2} \eta^{2} K_{0}(\kappa|\eta|) \\
& \left.-2\left(2+\kappa^{2} \eta^{2}\right) v^{2} \kappa|\eta| K_{1}(\kappa|\eta|)\right] \\
G_{z z}(\eta) & =-\frac{v E \kappa h}{\left(1-v^{2}\right) \pi} K_{1}(\kappa|\eta|)
\end{aligned}
$$

The plastic zone size $r_{p}$ can be determined so that the stress at the tip of the plastic zone is finite. This can be expressed mathematically as $K\left(a+r_{p}\right)=0$

Solution of the system of integral equations (7a) and (7b) can be obtained by using Gauss-Chebyshev quadrature method and applying an iterative procedure with respect to the plastic zone size. The results are shown in Fig.1 for three different ratios of crack size to plate thickness, together with the Dugdale model's solutions for plane-stress and plane-strain.

It is clear that for low applied stress (relative to material's yield stress) the present solutions do indeed recover the plane-strain solutions. However, at 
high applied stress $\left(\sigma_{y y}^{\infty} / \sigma_{Y S} \rightarrow 1\right)$, the plastic zone size approaches the planestress solution. Furthermore, in addition to the applied stress, the ratio of cracksize to plate thickness $h / a$ is another parameter affecting the extent of plastic zone.

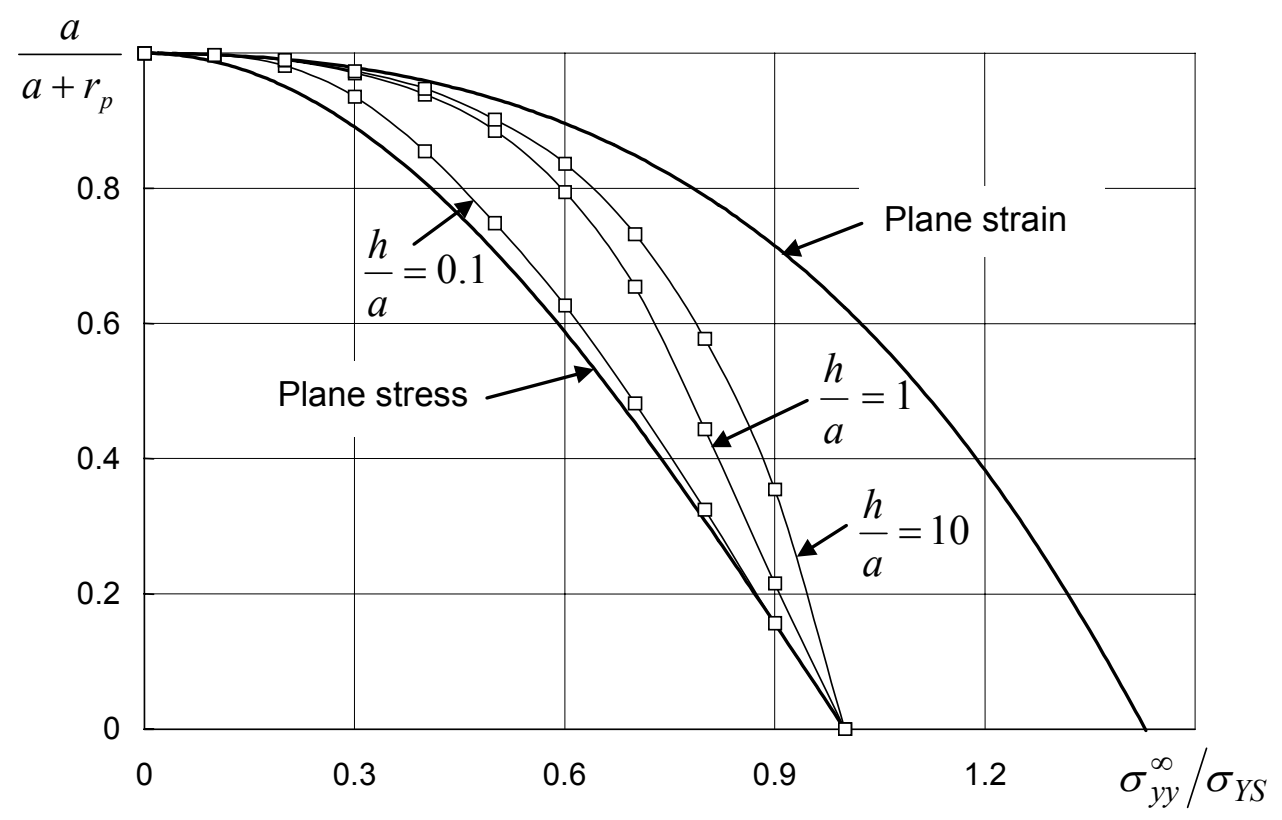

Fig.1 Plastic zone size as a function of the ration applied load to the yield load

\section{REFERENCES}

1. Broek, D. and Schijve, J. (1966) The influence of sheet thickness on crack propagation. Aircraft Engng, 38, 31-33.

2. Guo, W., Wang, C. H. and Rose, L. R. F. (1999). On the influence of cross sectional thickness on fatigue crack growth, Fatigue and Fracture of Engineering Materials and Structures, 22, 437-444.

3. Newman, J.C., Jr., Crews, J. H., Jr., Biglew, C. A. and Dawicke D. S. (1995) Variations of a global constraint factor in cracked bodies under tension and bending loading, Constraint Effects in Fracture Theory and Applications: Second Volume, ASTM STP 1244, M. Kirk and A. Baker eds., American Society for Testing and Materialsi, Philadelphia. 
4. O'Dowd, N. P. and Shih C. F. (1991) Family of crack-tip fields characterized by a triaxiality parameter: Part I-Structure of fields. J. Mech. Phys. Solids 39, 989-1015.

5. Huang Y. and Brocks, W. (1998) Quantification of constraint effects in elastic-plastic crack front fields, J. Mech. Phys. Solids, Vol.46, 219-241.

6. Brown M. W. and Miller K. J. (1985) Mode I fatigue crack growth under biaxial stress at room and elevated temperature, Multiaxial Fatigue, ASTM STP 853, 135-152.

7. Newman J. C., Jr. (1992) Effects of constraint on crack growth under aircraft spectrum loading, Fatigue of Aircraft Materials, Proc. of the Specialists' Conf. Dedicated to the 65th birthday of J. Schijve, Edit by A. Beukers, Th. de Jong, J. Sinke, A. Vlot and L. B. Vogelesang, Delft University Press, 83-109.

8. Wang, G. S. Palmberg B. and Blom A. F. (1992) Stress state-related fatigue crack growth under spectrum loading. Fatigue Fract. Engng. Mater. Struct., 15, 695-712.

9. Schijve J. (1987) Fatigue crack growth under vairable amplitude/spectrum loading, 3rd International Conference on Fatigue and Fatigue Thresholds, University of Virginia, Charlottesville, VA, USA, June 28-July 3.

10. Budiansky B. and Hutchinson J. W. (1978) Analysis of closure in fatigue crack growth. J. Appl. Mech. 45, 267-276.

11. Fleck N. (1986) Finite element analysis of plasticity induced crack closure under plane strain conditions, Engng Fract. Mech. 25, 441-449.

12. Newman J. C., Jr. and Bigelow C. A. (1993) Three-dimensional elasticplastic finite-element analyses of constraint variations in cracked bodies. Engng Fract. Mech. 46, 1-13.

13. Kane, T.R. and Mindlin, R.D. (1956). High frequency extensional vibrations of plates, Journal of Applied Mechanics, 23, 277-283

14. Yang, W. and Freund, L.B. (1985). Transverse shear effects for throughcracks in an elastic plate, Int. J. Solids and Structures, 21, 977-994.

15. Kotousov, A. and Wang, C. H. (2002). Three-dimensional stress constraint in an elastic plate with a notch, Int. J. Solids and Structures (in press).

16. Dugdale, D.S. (1960). Yielding of steel sheets containing slits. J. Mech. Phys. Solids 8, 100-104.

17. Hills, D.A., Kelly, P.A., Dai, D.N. and Korsunsky, A.M. (1996). Solution of Crack Problems, Kluwer Academic Publisher, Dordrecht. 